\title{
" Effet d'Utilisation des Histoires Numériques sur le Développement Des compétences de la Compréhension Orale en Français Chez Les Elèves du Cycle primaire Aux Ecoles Des Langues"
}

\section{Dr. Mahmoud Ezz El-Arab El-Sayed}

\section{Abstrait :}

Le problème de cette recherche se manifeste dans la faiblesse des compétences de la compréhension orale chez les élèves de la cinquième année primaire. Cette faiblesse peut être revenir à l'utilisation des stratégies d'enseignement inconvenables à leurs styles d'apprentissage avec la négligence des supports technologiques. Notre étude actuelle essaie de répondre aux sous-questions suivantes- 1 Quelles sont les compétences de la compréhension orale nécessaires aux élèves de la cinquième année primaire aux écoles des langues-९Jusqu'à quel point les élèves de la cinquième année primaire aux écoles des langues -ils possèdent ces compétences ?Quelle est la représentation suggérée pour utiliser les histoires numériques afin de développer les compétences de la compréhension orale en Français chez Les élèves du cycle primaire aux écoles des langues - Quel est l'effet d'utilisation des histoires numériques sur le développement des compétences de la compréhension orale en Français chez Les élèves de la cinquième année primaire aux écoles des langues ¿La recherche actuelle a abouti que Les histoires numériques ont développé les compétences de la compréhension orale chez les élèves du cycle primaire grâce à un climat d'encouragement et de motivation qui contribue à exprimer librement et de comprendre soigneusement la langue parlée. Autrement les techniques des histoires numériques ont favorisé les occasions d'interaction entre les élèves à travers des groupes. Cette interaction leur permet échanger leurs expériences et leurs connaissances en supportant les compétences de la compréhension orale.

Mots - clés : $\quad$ Les histoires numériques - la compréhension orale la narration - les multimédias - l'interaction - l'expression orale la communication - les images - les vidéos courts - le commentaire - l'imagination - l'apprentissage amusé - la motivation.

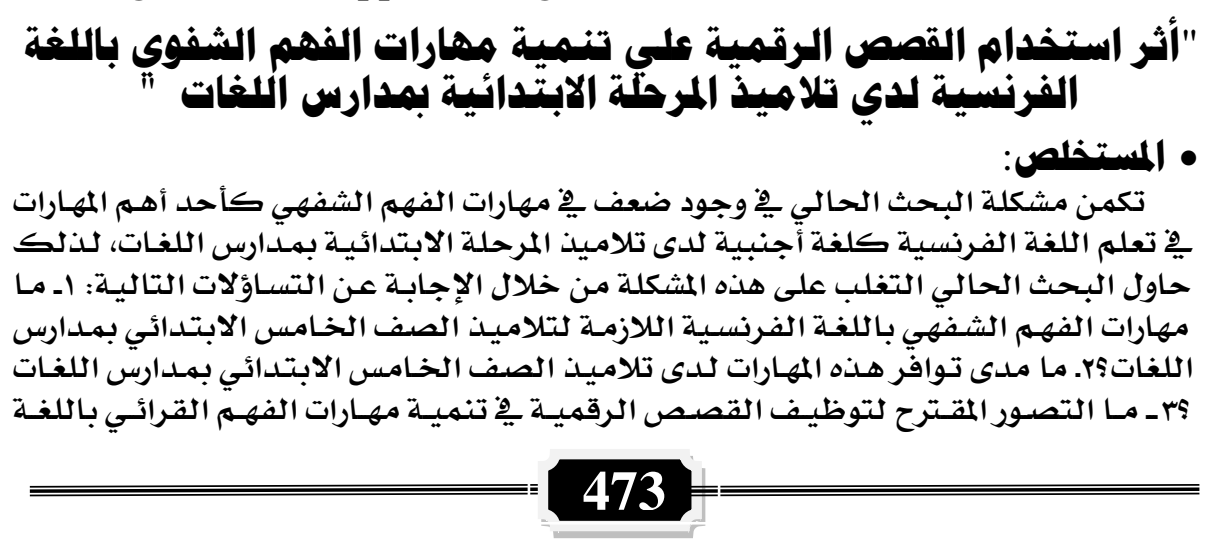




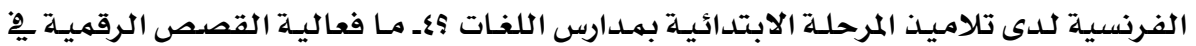

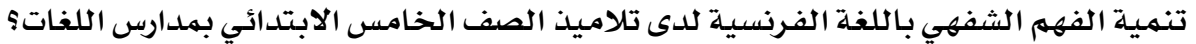

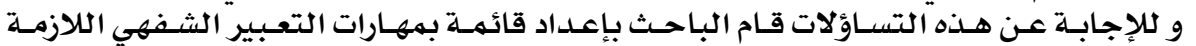

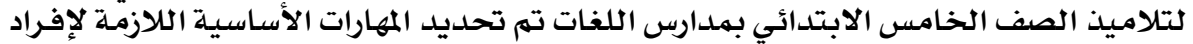

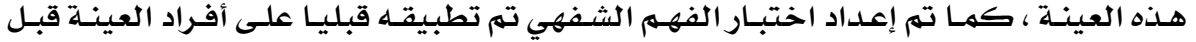

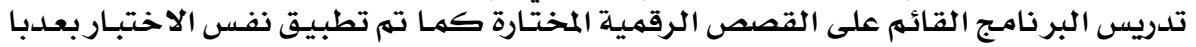

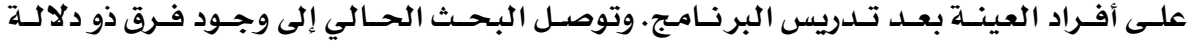

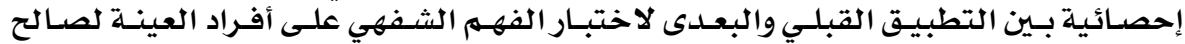

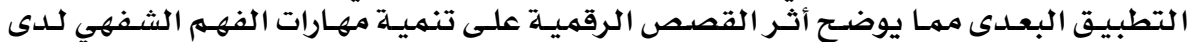

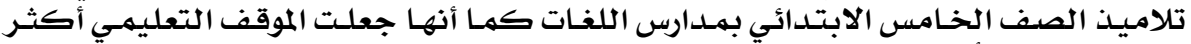

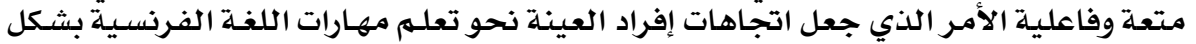

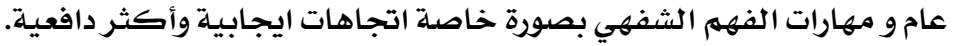

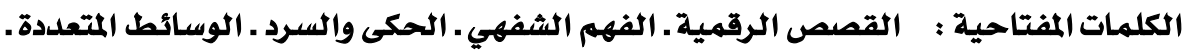

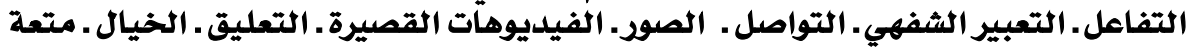

\section{Introduction :}

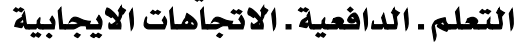

Pour Enseigner une langue, il faut maîtriser les savoirsfaires nécessaires : exprimer et comprendre des notions, satisfaire un certain nombre des fonctions de communication, utiliser et comprendre des actes de langage. Le but fondamental est de rendre l'homme capable de comprendre des textes oraux et écrits dans des conditions normales de communication et, ultérieurement, d'en produire en puissant dans les modèles de tous les ordres qu'il aura intégrés.

Pareillement, les apprenants d'une langue étrangère se trouvent confrontés immédiatement à la langue orale dès le début de leur apprentissage et souhaitent être, le plus rapidement possible, capables de communiquer oralement et donc acquérir une compétence d'expression et de compréhension.

Dans l'enseignement/apprentissage des langues étrangères, les activités de production orale, production écrite et de compréhension écrite sont très importantes et primordiales mais celles de compréhension orale se placent davantage au coeur des apprentissages parce que tout d'abord, l'écoute est la compétence la plus exercée dans le

\section{4}




\section{Number 57 , Part 2 , January, 2015}

quotidien et dans le contexte scolaire.(Aslım-Yetiş, 2012 : 34)

Les travaux sur la communication montrent que les deux composantes oral/écrit et expression/compréhension sont en étroite corrélation et qu'il est difficile de les dissocier. L'apprentissage de l'une sert à développer l'autre : bien lire implique bien écrire, bien entendre et écouter, bien parler implique bien s'exprimer et comprendre.

La compréhension orale est une des étapes les plus fondamentales de la communication et dans l'acquisition d'une langue étrangère. L'écoute dans des situations authentiques permet à l'élève de se familiariser avec les sons de la langue non maternelle, elle permet de présenter à l'apprenant des discours plutôt que des phrases, elle amène l'élève à s'appuyer sur les éléments du texte et les connaissances personnelles pour comprendre un message oral. (Ferroukhi, 2009 : 275)

La compréhension orale est définie comme la capacité à comprendre à partir de l'écoute d'un énoncé ou d'un document sonore. Autrement dit, les élèves maitrisent la compréhension orale quand ils comprennent ce qui leur a été lu ou dit. (Belair, 2012 : 11)

Par ailleurs, l'activité de compréhension orale est une compétence qui pose des problèmes et difficultés particuliers : (1) il ne s'agit pas d'une activité contrôlable par l'individu comme, par exemple, en production orale où « le sujet est maître de son propre rythme et [où] il décide librement aussi du contenu de ce qu'il veut dire. » Ou bien, comme en compréhension écrite où l'apprenant peut lire à son rythme, s'attarder sur les mots difficiles, réfléchir tant qu'il le souhaite tout comme il le peut en expression écrite. Or, en compréhension orale, il est question d'un contenu qui « coule », qui avance selon sa cadence et qu'il faut pourtant réussir à écouter, comprendre afin de donner très vite une

\section{5}




\section{Number 57 , Part 2 , January, 2015}

rétroaction pour que l'échange continue. (Porcher, 2005 : 46).

La compréhension orale n'est cependant pas un traitement soit descendant soit ascendant, mais bien un processus d'interprétation interactif au cours duquel les auditeurs font appel à la fois à leurs acquis et à leurs connaissances linguistiques pour comprendre le message. Selon Rost la fréquence à laquelle les auditeurs utilisent un processus plutôt qu'un autre va dépendre de leurs connaissances de la langue, du degré auquel le sujet leur est familier ou du but de l'écoute. (Rost, 2002 : 35)

La compréhension orale déplaît à beaucoup d'apprenants en classe et crée de l'anxiété langagière en eux. Cette anxiété qui est une "sensation de tension et d'appréhension associée aux contextes dans lesquels la langue étrangère est utilisée, y compris la production orale, la compréhension orale et l'apprentissage1. (Harmer, 2007: 304).

Si la compréhension de l'oral semble difficile à acquérir pour les élèves, c'est parce qu'elle est rarement pratiquée en classe. Pourtant, c'est une activité indispensable dans l'apprentissage d'une langue. Cette compétence se construit petit à petit grâce à l'écoute d'une diversité de messages (contes, chansons, consignes), dans des situations de communication variées. (Rost, 2002 : 35)

D'ici, il faut introduire une pédagogie de l'écoute pour apprivoiser l'oreille des apprenants, non seulement au niveau de la prosodie1, mais également au niveau linguistique (enrichissement du vocabulaire, mémorisation de structures de phrases). Il faut également favoriser le plus possible l'exposition à la langue étrangère et aux différents types de documents. (Masuy \& Genevieve, 2010 : 36)

Par rapport, L'enseignement et l'apprentissage du français langue étrangère au niveau du cycle primaire vise développer aisément chez les jeunes apprenants les 


\section{Number 57 , Part 2 , January, 2015}

capacités de communiquer soit par l'expression ou soit par la compréhension à travers des situations scolaires ou extrascolaires qui correspondent à leur développement cognitif. (Taghezout, $2009: 4$ )

En didactique de français langue étrangère FLE, l'oral soit l'expression ou la compréhension a toujours fait partie, d'une manière ou d'une autre, des pratiques d'enseignement : lecture à haute voix, conversation, dialogue entre apprenants, récitation, élocution, etc. Mais la pratique de l'oral cherche encore une véritable place dans l'enseignement. Les enseignants peinent encore à considérer l'oral comme objet d'enseignement à part entière. C'est peut-être la raison pour laquelle il fait davantage l'objet de recherches depuis quelques années.

C'est au début du 20ème siècle que la technologie commence à être utilisée en didactique grâce à l'électricité et à l'utilisation des moyens qu'elle permet. La possibilité de capter le son et, par la suite, de le reproduire ainsi que la possibilité de faire de même avec l'image, d'abord fixe puis animée, ont tout de suite intéressé tant les méthodologues que les pédagogues qui ont théorisé et expérimenté l'usage de ces nouveaux supports pour l'enseignement. (Defay et Deltour, 2003 : 263)

L'évolution technique a été lente pendant des siècles et n'a donc pas nécessité de compétences techniques développées. Mais depuis la seconde moitié du 20ème siècle, l'accélération des progrès techniques met en demeure l'enseignant d'accéder à de nouveaux savoir-faire, le plus souvent éloignés de sa formation d'origine. Ces technologies nouvelles ne sont pas que de simples changements de supports. Elles impliquent le renouvellement des pratiques d'enseignement, la remise en question des rapports entre l'enseignant, l'objet d'enseignement et les apprenants.

Avec le multimédia on passe de technologies qui offraient la possibilité de développer en priorité une seule 


\section{Number 57 , Part 2 , January, 2015}

compétence, orale ou écrite, à des technologies qui rapprochent plus de la multi analité de la communication réelle. Il permet, de plus, un enseignement-apprentissage du FLE non seulement d'un point de vue linguistique mais également dans les usages culturels de la langue cible. Le multimédia, enfin et surtout, transforme profondément les rapports entre l'enseignant, les apprenants et le savoir. De dispensateur du savoir il devient guide vers le savoir. (Ginet, 2007 : 169)

L'utilisation de documents authentiques en classe de langue étrangère n'a d'intérêt que si elle est inclue au sein d'un programme méthodologique d'enseignementapprentissage du FLE précis et cohérent et que si ces documents sont exploités dans leurs qualités spécifiques, d'où la nécessité, d'une part, d'établir des stratégies d'exploitation respectant les situations de communication que ces documents véhiculent et, de l'autre, d'essayer de restituer l'authenticité de leurs réceptions. (Viallon, 2002 : 211)

L'utilisation de la technologie et de l'ordinateur dans l'acquisition d'une langue seconde ou étrangère favorise un facteur primordial pour faciliter le développement des compétences compréhension oral, auditive, l'expression orale, la compréhension de lecture et l'expression écrite. (Watt, 2002 : 40)

L'histoire numérique est une des modernes technologies que l'enseignant peut l'employer dans la classe comme un changement important dans le domaine de l'éducation. Elle fournit aux apprenants par les programmes d'ordinateur graphiques avec des grandes couleurs, des mouvements et des effets acoustiques. (Green, 2011, 7)

D'ici, L'histoire numérique se représente une façon dont les enseignants peuvent utiliser la technologie pour intervenir des méthodes pédagogiques innovantes

\section{8}




\section{Number 57 , Part 2 , January, 2015}

d'enseignement. La narration numérique implique la fonction des ressources numériques dans les environnements d'apprentissage pour produire des récits multimédias.

Dans cet égard, Frazel (2010 : 125) énumère les avantages des histoires numériques comme suit :

- Elles favorisent aux apprenants une bonne occasion pour acquérir des réelles expériences éducatives ou des informations variées.

- Elles fournissent aux apprenants des situations d'apprentissage plus interactives.

- Elles permettent aux apprenants d'apprendre individuellement ou collectivement (en équipe).

- Elles se représentent un facteur vital pour comprendre, produire et communiquer grâce aux images visuelles.

- Elles peuvent promouvoir la créativité et la résolution de problèmes tout en encourageant l'autonomie et l'initiative personnelle.

L'étude actuelle rejoint ces préoccupations. Elle a pour objectif d'utiliser les histoires numériques pour développer les compétences de la compréhension orale.

\section{Position du Problème :}

La didactique de l'oral soit (exprimer ou comprendre) dans les pays francophones demeure la compétence langagière la moins considérée dans l'enseignement du français à cause des diverses facteurs comme l'absence de matériel didactique Claire ; l'utilisation des méthodes d'enseignement intuitives ; les difficultés liées à la situation de présentation de l'activité orale et notamment à la réaction du pairs et à l'environnement dans le quel se produit l'activité. (La Fontaine et al. ,2007,49)

D'autre part, les résultats de beaucoup d'études comme (Carette 2001, Harmer 2007, Ferroukhi 2009 et Alrabadi 2010) ont assuré que L'enseignement et l'apprentissage du

\section{9}




\section{Number 57 , Part 2 , January, 2015}

français langue étrangère au niveau du cycle primaire vise à développer quatre savoirs faires (deux compréhensions orale et écrite, et deux expressions orale et écrite). Malgré que l'importance de l'enseignement et du développement des compétences de la compréhension orale chez les jeunes apprenants, cette compétence est toujours négligée dans la didactique du Français comme langue étrangère. La maitrise de la langue orale soit (compréhension ou expression) est non seulement une nécessité urgente dans la vie quotidienne mais aussi une des conditions fondamentales de l'intégration sociale.

En outre, pour confirmer les résultats des études antérieures, on a appliqué une étude pilote déclarative non standardisée à 35 élèves de la cinquième année primaire d'une d'écoles des langues à Damiette pour l'année scolaire 2014 - 2015. Cette étude est un test de compréhension orale composant de trois questions principales qui nécessitent une performance orale à l'égard des élèves de l'échantillon.

Les résultats de cette étude pilote ont montré l'existence d'une faiblesse tangible dans les compétences de la compréhension orale chez la plupart des élèves de l'échantillon. 31 élèves ne peuvent pas performer les compétences de la compréhension orale demandées.

De telle sorte que l'étude actuelle essaie d'employer les histoires numériques pour développer les compétences de la compréhension orale chez les élèves du cycle primaire.

\section{Problématique de la recherche :}

A la lumière des résultats des études antérieures et de l'étude pilote, le problème de cette recherche se manifeste dans la faiblesse des compétences de la compréhension orale chez les élèves de la cinquième année primaire. Cette faiblesse peut être revenir à l'utilisation des stratégies d'enseignement inconvenables à leurs styles d'apprentissage avec la négligence des supports technologiques.

\section{0}




\section{Number 57 , Part 2 , January, 2015}

L'étude actuelle essaie de vérifier l'effet d'utiliser les histoires numériques sur le développement des compétences de la compréhension orale chez les élèves de la cinquième année primaire aux écoles des langues.

Notre étude actuelle essaie de répondre à la question principale suivante : "Quel est l'effet d'Utilisation des histoires numériques sur le développement des compétences de la compréhension orale en Français chez Les élèves du cycle primaire aux écoles des langues " ?

Il s'agit donc de répondre aux sous-questions suivantes:

1. Quelles sont les compétences de la compréhension orale nécessaires aux élèves de la cinquième année primaire aux écoles des langues?

2. Jusqu'à quel point les élèves de la cinquième année primaire aux écoles des langues -ils possèdent ces compétences?

3. Quelle est la représentation suggérée pour utiliser les histoires numériques afin de développer les compétences de la compréhension orale en Français chez Les élèves du cycle primaire aux écoles des langues "

4. Quel est l'effet d'utilisation des histoires numériques sur le développement des compétences de la compréhension orale en Français chez Les élèves de la cinquième année primaire aux écoles des langues?

\section{Objectifs de la recherche ;}

La recherche actuelle a pour objectif de :

- Développer les compétences de la compréhension orale en Français chez les élèves de la cinquième année primaire aux écoles des langues.

- Vérifier l'effet d'utilisation des histoires numériques sur le développement des compétences de la langue française en général et des compétences de la compréhension orale surtout.

\section{Importance de la recherche :}

Cette recherche peut contribuer à :

\section{1}




\section{Number 57 , Part 2 , January, 2015}

- Dégager les différentes compétences de la compréhension orale en Français nécessaires aux élèves du cycle primaire dans les écoles des langues.

- Rendre les enseignants plus attentifs et conscients des nouvelles stratégies et des styles d'apprentissage convenables pour la compréhension orale.

- Montrer l'importance pédagogique des histoires numériques et leur rôle dans le développement des compétences langagières généralement et la compréhension orale particulièrement.

- Présenter aux enseignants du Français un test pour évaluer les compétences de la compréhension orale chez les élèves.

- Ouvrir de nouveaux domaines à d'autres recherches portant sur les histoires numériques pour développer les autres compétences langagières.

\section{Limites de la recherche:}

La présente recherche se limite à :

- Un échantillon d'élèves de la cinquième année primaire d'une d'écoles des langues à Damiette.

- Quelques compétences de la compréhension orale nécessaires aux élèves de la de la cinquième année primaire.

\section{Instruments de la recherche :}

- Une liste des compétences de la compréhension orale nécessaires aux d'élèves de la cinquième année primaire.

- Un pré-post test pour évaluer les compétences de la compréhension orale chez les élèves de l'échantillon.

- Quelques unités proposées pour employer les histoires numériques afin de developper les compétences de la compréhension orale choisies.

\section{Hypothèses de la recherche :}

La recherche actuelle va essayer d'examiner les hypothèses suivantes :

- Il y a une différence statistiquement significative entre les moyennes des notes du groupe expérimental au pré et

\section{2}




\section{Number 57 , Part 2 , January, 2015}

post-test des compétences de la compréhension orale en faveur du post-test.

- Les histoires numériques ont un effet pour développer les compétences de la compréhension orale.

\section{Plan de la recherche :}

Pour répondre aux questions précédemment citées, nous avons procédé de la manière suivante :

- Etude théorique réflexive des études antérieures et des ouvrages (arabes et étrangers) portant sur :

A. la définition de la compréhension orale, ses objectifs, ses compétences et les approches favorables à la compréhension orale.

B. l'origine des histoires numériqus, leur importance, leurs caractéristiques et leurs techniques.

- Elaboration d'une liste des compétences de la compréhension orale et la presenter au jury pour limiter les competences nécessaires aux élèves de la cinquième année primaire.

- Préparartion et standardisation du pré-post test pour détecter à quel point les élèves maîtrisent-ils les compétences de la compréhension orale.

- Elaboration des unités proposées à la lueur de la philosphie des histoires numériques en suivant quelques démarches :

A. définir les objectifs généraux et spécifiques des unités.

B. préciser les sources du des unités.

C. préparer le contenu du des unités.

D. délimiter la stratégie proposée de l'enseignement, les activités, les exercices et les moyens d'évaluation.

E. Présenter les unités proposées au jury et parvenir à sa forme finale.

- Choisir un groupe expérimental d'élèves de la de la cinquième année primaire d'une d'écoles des langues à Damiette. 


\section{Number 57 , Part 2 , January, 2015}

- Application du pré-test des compétences de la compréhension orale aux membres de l'échantillon.

- Enseignement des unités proposées au groupe expérimental, puis application du post-test des compétences de la compréhension orale aux membres de l'échantillon.

- Vérification des hypothèses, analyse et interprétation des résultats.

- Formulation des recommandations et offert des suggestions de la recherche.

\section{Terminologie de la recherche :}

Pour définir cette notation, nous avançons d'abord certaines définitions qui ont paru dans des études antérieures, puis nous donnons notre propre définition.

\section{Les histoires numériques :}

- L'histoire numérique est l'art de combiner la narration avec les médias numériques tels que les images, les sons et les vidéos pour créer une histoire courte. (Robin, 2006 : 99)

- L'histoire numérique est considéré comme une expression moderne de l'art ancien du conte. Tout au de l'histoire, la narration a été utilisée pour partager les connaissances, la sagesse et les valeurs. Les histoires numériques sont adaptées à chaque milieu successive qui a vu le jour, à partir du cercle du feu de camp à l'écran argent et maintenant l'ordinateur écran. (Robin, 2008 : 490)

- L'histoire numérique est une conte qui prose l'action réaliste ou imaginaire en basant sur l'utilisation d'un des programmes d'intégrer les multimédias pour exposer les photos, les cartes, les textes, le background musique, les commentaires audio et les clips vidéo afin de présenter les événements, les personnalités, les attitudes, les phénomènes. (Hussein Abdel Bassat, 2010 : 200)

Dans le cadre des définitions précédentes, le chercheur peut définir les histoires numériques comme suit :

\section{4}




\section{Number 57 , Part 2 , January, 2015}

C'est une nouvelle création à la narration qui fonctionne les multimédias comme la photo, les cartes, les textes, la musique, la voix, le vidéo et les commentaires pour exposer une conte soit réaliste ou imaginaire en réalisant des objectifs cibles.

\section{La compréhension orale :}

- La compréhension orale consiste à intégrer une connaissance nouvelle aux connaissances existantes en s'appuyant sur les paroles ou le texte, i.e. ce qu'on appelle aussi entrée ou stimulus. (Ferroukhi, 2009 : 273)

- La compréhension orale suppose la connaissance du système pédagogique ou graphique et textuel, la valeur fonctionnelle et sémantique des structures linguistiques véhiculées, mais aussi la connaissance des règles socioculturelles de la communauté dans laquelle s'effectue la communication, sans oublier les facteurs extralinguistiques qui interviennent notamment à l'oral comme les gestes, les mimiques ou tout autre indice qu'un locuteur utilise inconsciemment. (Alrabadi, 2010 : 18)

- La compréhension orale est définie comme la capacité à comprendre à partir de l'écoute d'un énoncé ou d'un document sonore. Autrement dit, les élèves maîtrisent la compréhension orale quand ils comprennent ce qui leur a été lu ou dit. Cette capacité met en jeu différentes opérations mentales : se repérer dans ce qu'on entend en distinguant les sons, les mots, les phrases; repérer les informations apportées ; sélectionner celles qui sont importantes. (Belair, 2012 : 11)

Dans le cadre des définitions précédentes, le chercheur peut définir la compréhension orale comme suit :

La reconnaissance du sens d'un discours. La compréhension orale est donc la capacité d'accéder au sens à partir de l'écoute, d'un énoncé ou d'un document sonore. 


\section{Number 57 , Part 2, January, 2015}

Elle suppose la connaissance du système de prononciation ainsi que des règles linguistiques et socioculturelles.

\section{1- L'Etude théorique}

Dans notre étude théorique, nous essayons de traiter ce sujet dans deux parties suivantes :

- La première partie est intitulée par "la compréhension orale" qui implique les points suivants :

- La nature de la compréhension orale.

- Les facteurs qui influent sur la compréhension orale.

- Les stratégies et les approches utilisées pour améliorer la compréhension orale.

- La deuxième partie est intitulée par "les histoires numériques" qui implique les points suivants :

- La définition des histoires numériques

- Les avantages des histoires numériques.

- Les types des histoires numériques.

- Les élèments de l'histoire numérique.

- Les étapes de la préparation de l'histoire numérique.

- Les applications pédagogiques des histoires numériques.

I- La première partie : La compréhension orale

I.1 La nature de la compréhension orale

Dans l'enseignement/apprentissage des langues étrangères, les activités de production orale, production écrite et de compréhension écrite sont très importantes et primordiales mais celles de compréhension orale se placent davantage au cour des apprentissages parce que tout d'abord, l'écoute est la compétence la plus exercée dans le quotidien et dans le contexte scolaire. Comme le dit Cornaire \& Raymond (1999 : 200) nous passons « 45\% de note temps à écouter, contre $30 \%$ à nous exprimer oralement, $16 \%$ à lire et $9 \%$ à écrire. »

D'ici, l'écoute est à la base du travail de la compréhension orale. Il faut savoir qu'en début d'apprentissage d'une langue étrangère, il est toujours difficile pour les élèves d'écouter et de comprendre. En effet, les élèves ont du mal à distinguer les sons français, dont certains n'existent pas dans leur

\section{6}




\section{Number 57 , Part 2 , January, 2015}

langue maternelle. Ils ont également des difficultés pour distinguer les mots et comprendre les phrases. Faire des écoutes régulières en classe habituera les élèves à se familiariser avec les sons, les mots et les phrases en français. À force de pratiquer ces écoutes, les élèves deviennent alors plus habiles aux tâches de compréhension et leur compétence langagière s'améliore. (Maurer, 2001 : 29)

En psychologie, comprendre consiste à intégrer une connaissance nouvelle aux connaissances existantes en s'appuyant sur les paroles ou le texte. Ce qu'on appelle aussi entrée ou stimulus. (Gremmo et Holec, 1990 : 280)

Selon D.Gaonac'h, " la perception ou la compréhension est possible grâce à un processus d'assimilation, il s'agit de construire une représentation de L'information dans les termes des connaissances antérieurement acquises ». Il ajoute que "les processus de réception du langage sont constitués de cycles d'échantillonnage, prédiction, test et confirmation ». (Gaonac'h, $1990: 30$ )

Alors, Comprendre est la reconnaissance du sens d'un discours. La comprehension orale est donc la capacité d'accéder au sens à partir de l'écoute, d'un énoncé ou d'un document sonore. Elle suppose la connaissance du système de prononciation ainsi que des règles linguistiques et socioculturelles. (NONNON, $2000: 80$ )

Ainsi, La compréhension orale est définie comme la capacité à comprendre à partir de l'écoute d'un énoncé ou d'un document sonore. Autrement dit, les élèves maîtrisent la compréhension orale quand ils comprennent ce qui leur a été lu ou dit. Cette capacité met en jeu différentes opérations mentales : se repérer dans ce qu'on entend en distinguant les sons, les mots, les phrases; se repérer les informations apportées ; sélectionner celles qui sont importantes. (Belair, $2012: 11)$ 


\section{Number 57 , Part 2 , January, 2015}

Autrement, la compréhension orale est une compétence qui vise à faire acquérir progressivement à l'apprenant des stratégies d'écoute premièrement et de compréhension d'énoncé à l'orale, deuxièmement. Il ne s'agit pas de tout faire comprendre aux apprenants, qui ont tendance à demander une définition pour chaque mot. L'objectif est exactement inverse ; il est question, au contraire, de former nos auditeurs à devenir plus sûrs d'eux, plus autonomes progressivement. (FERROUKHI, 2009 : 275)

Donc, la compréhension orale s'intéresse à favoriser l'écoute et la compréhension d'un grand nombre de messages, peu importe leur source, le débit et la voix de celui qui parle.

D'autre part, LAFONTAINE (2001 : 176- 177) voit que la compréhension orale dépendra de la relation entre trois composantes du processus de compréhension. La relation optimale entre ces composantes favorisera, chez l'élève, une meilleure compréhension à l'oral; ces composantes sont comme suit:

\section{Le récepteur:}

Trois facteurs décrivent le récepteur dans ce processus : ses connaissances sur la langue et sur le monde qui l'entoure, ses attitudes face à l'écoute en général et face à un discours en particulier et ses habiletés de mise en oeuvre du processus. L'enseignement et l'apprentissage de l'écoute préconisés dans le programme d'études - document de mise en oeuvre visent à bâtir sur les connaissances de l'élève, à développer ses habiletés de mise en oeuvre et à consolider ses attitudes afin de l'amener à une autonomie de plus en plus grande en écoute.

\section{Le discours oral:}

La composante "discours oral" porte sur le message à écouter et regroupe trois éléments : l'intention de l'émetteur, l'organisation du discours et le contenu. Ces

\section{8}




\section{Number 57 , Part 2 , January, 2015}

trois éléments interagissent dans un discours oral pour véhiculer un message particulier. L'habileté à dégager l'intention de l'émetteur aidera l'élève à reconnaître la structure générale du discours déterminant l'organisation des idées et, également à reconnaître le contenu délimitant les concepts, les connaissances et le vocabulaire. L'enseignement et l'apprentissage de l'écoute préconisés dans le programme d'études - document de mise en oeuvre visent à outiller l'élève dans ce domaine en le mettant en contact avec une variété de discours oraux et diverses formes de communication orale.

\section{Le contexte:}

Les éléments qui composent le contexte ne concernent pas le discours oral en lui-même mais influent sur la compréhension de celui-ci. La composante contexte regroupe toutes les conditions auxquelles l'élève est exposé lorsqu'il prend contact avec le discours oral. Il existe trois contextes :

Le contexte psychologique qui inclut l'intention d'écoute et l'intérêt pour le discours ; le contexte social qui regroupe les interventions de l'enseignant et des pairs ; le contexte physique dans lequel on retrouve le temps disponible et le bruit. La relation optimale entre le récepteur, le discours oral et le contexte résultera en une meilleure compréhension du discours.

Pour Carette (2001) « l'écoute orientée est constitutive de la compréhension orale » c'est-à-dire pour mieux écouter, il faut apprendre à faire varier sa façon d'écouter en fonction d'un objectif de compréhension. (Carotte, 2001: 129)

Parallèlement, Soussi et Martin (1999) mettent en lumière la même idée en indiquant qu'il y a différents types d'écoute qui sont mis en œuvre, en fonction de l'objectif de compréhension :

- écoute sélective : apprendre à n'écouter que le(s) passage(s) qui est (sont) nécessaire à la réalisation d'une tâche, apprendre à ', ne pas entendre'" le reste.

\section{9}




\section{Number 57 , Part 2 , January, 2015}

- écoute détaillée : apprendre à prendre connaissance de tout ce qu'on veut écouter (dans un passage particulier, dans une catégorie d'informations, dans un discours oral). C'est une écoute exhaustive, de durée variable.

- écoute globale : apprendre à découvrir suffisamment d'éléments du discours pour en comprendre la signification générale.

- écoute réactive : apprendre à utiliser ce qu'on comprend pour faire quelque chose (prendre des notes, réaliser un gâteau, faire fonctionner un appareil, etc.). Ce type d'écoute nécessite de savoir mener deux opérations en même temps : il faut par exemple décider qu'elles informations sont importantes, décider si l'auditeur doit intervenir sur le discours du locuteur (si l'interaction est possible), etc., tout en continuant à écouter.

- écoute de veille : écoute automatique, sans réelle compréhension, mais qui fait place à une autre écoute dès qu'un mot ou groupe de mots déclenche un intérêt pour le discours.

Ces différents types d'écoute peuvent éventuellement se succéder dans une situation de compréhension et requièrent alors la mise en œuvre de véritables stratégies d'écoute.

Dans le même cadre, Séverine (2000, 34-35) montre aussi qu'il existe trois niveaux de compréhension en écoute : la compréhension littérale, la compréhension interprétative et la compréhension critique. Chacun de ces trois niveaux travaille des habiletés spécifiques reliées à un niveau particulier du processus de compréhension.

\section{La compréhension littérale:}

L'élève, lorsqu'il travaille la compréhension littérale du discours oral, repère des informations, des idées, des situations exprimées explicitement dans le discours. Les réponses à son questionnement intérieur sont mentionnées explicitement dans le message oral et les indices qui établissent la relation entre la question et la réponse sont clairement exprimés.

\section{0}




\section{Number 57 , Part 2 , January, 2015}

\section{Exemple :}

- Discours : En fin de semaine, je suis allé à la pêche. Mon père a gagné un trophée car il a pêché le plus gros poisson.

- Question: Pourquoi le père a-t-il gagné un trophée?

- Réponse: Parce qu'il a pêché le plus gros poisson.

\section{La compréhension interpretative :}

L'élève, lorsqu'il travaille la compréhension interprétative, est appelé à faire des Inférences puisque la réponse à son questionnement intérieur, quoique énoncée dans le discours oral, est implicite. Sa question et sa réponse découlent toutes les deux du message oral mais il n'y a pas d'indice grammatical qui relie la question et la réponse; l'élève doit l'inférer. Les éléments du paralangage et les éléments de prosodie sont une composante du discours oral dont l'élève doit interpréter la Signification. Ces éléments ont un lien direct avec le message transmis mais leur sens est toujours implicite et doit être interprété par l'élève.

\section{Exemple :}

- Discours : Je suis un inventeur de jouets. Parmi mes inventions, on retrouve la toupie, le yoyo et le jeu de cubes. La toupie et le jeu de cubes se vendent très bien.

- Question : Quel jouet n'est pas un succès?

- Réponse : Le yoyo car il ne se vend pas bien.

\section{La compréhension critique}

L'élève, lorsqu'il travaille la compréhension critique, est appelé à réagir à son questionnement intérieur en s'appuyant sur ses connaissances des formes de discours, des structures textuelles et des indices donnés par l'émetteur pour susciter des réactions affectives ou intellectuelles. Quoique les éléments de sa réponse pourraient se retrouver dans le message oral, les réponses n'apparaissent ni explicitement, ni implicitement puisqu'elles ont comme point de départ les réactions personnelles de l'élève. L'élève utilisera également les éléments du paralangage et les

\section{1}




\section{Number 57 , Part 2 , January, 2015}

éléments de prosodie contenu dans le discours oral pour établir des liens entre ses réactions personnelles et les parties du discours sur lesquelles portent ses réactions.

\section{Exemple:}

- Discours : La vie a beaucoup changé depuis 20 ans. Nous sommes beaucoup plus conscients du rôle que nous pouvons jouer pour préserver l'environnement. Question : Expliquez pourquoi on ne se préoccupait pas de l'environnement il y a20 ans?

- Réponse : Les réponses varieront selon les élèves.

Alors, il est clair que la compétence de réception orale est la plus difficile à acquérir et la plus indispensable. Son absence met l'apprenant dans une sorte d' " insécurité linguistique ».Or, en termes d'évaluation, la compétence « écouter » est « la mal aimée » et est « souvent minorée » dans les pratiques de classe (Veltcheff et Hilton, 2003 : 80)

Enfin, on peut dire que la compréhension orale est donc la capacité d'accéder au sens à partir de l'écoute, d'un énoncé ou d'un document sonore. Elle suppose la connaissance du système de prononciation ainsi que des règles linguistiques et socioculturelles.

\section{I.2 Les facteurs qui influencent sur la compréhension orale d'une langue étrangère}

Il existe de nombreux facteurs qui influent sur la compréhension orale. Il y a plusieurs facteurs qui jouent un grand rôle dans la compétence de la compréhension orale, Parmi ces facteurs, il y a ceux qui aident et d'autres qui entravent l'accès au sens. EMBARKI (2012) énumère ces facteurs comme suit :

\section{I.2.1 Le debit :}

Charles et Williame définissent le débit comme : «La vitesse à laquelle l'orateur parle. " (Charles \& Williame, 1997: 4)

\section{2}




\section{Number 57 , Part 2, January, 2015}

Le débit c'est aussi la vitesse d'articulation c'est-à-dire le nombre de phonèmes ou de syllabes articulés dans une seconde. La vitesse joue un rôle important dans la compréhension orale. Il faut donc faire attention à cette question et être prudent concernant les seuils de débit proposés par certains chercheurs, c'est-à-dire la vitesse à laquelle la compréhension commence à diminuer.

En conclusion, quand le débit d'un texte augmente la compréhension diminue, alors, il faut continuer à parler aux élèves à une vitesse normale.

\subsubsection{Les pauses et les hésitations :}

La pause correspond à un arrêt définitif ou à une suspension de la parole pour un Court laps de temps et elle peut aussi marquer la fin d'un groupe rythmique. Les pauses faciliteraient la compréhension, en ménageant du temps pour traiter l'information et en permettant de regrouper les unités en constituants.

Les chercheurs ont montré que des pauses d'une durée de trois secondes qui segmentent le texte ont apporté une aide précieuse à la compréhension, de l'autre part d'autres chercheurs ont mis en cause ces expériences en donnant comme argument qu'au contraire, les pauses et les hésitations constituent des sources d'erreurs pour l'apprenant en langue étrangère.

\subsubsection{Le décodage auditif :}

Concernant le décodage auditif, il faut savoir que certaines étapes sont indispensables pour arriver au stade de la compréhension. Ces étapes sont : audition, perception et traitement de l'information par le cerveau.

La transformation des sons en éléments de signification s'appuie sur la différenciation hémisphérique du cerveau. En effet, l'hémisphère droit est Plus spécialisé dans le traitement de la musique, de l'intonation des émotions alors que la gauche traite surtout les éléments linguistiques, phonétiques et cognitifs du langage. Les deux hémisphères

\section{3}




\section{Number 57 , Part 2 , January, 2015}

fonctionnent toutefois en étroite relation et se rendent mutuellement service, par exemple, en compensant en cas d'insuffisance de l'un d'eux. La gauche traite surtout les éléments linguistiques, phonétiques et cognitifs du langage. Les deux hémisphères fonctionnent toutefois en étroite relation et se rendent mutuellement service, par exemple, en compensant en cas d'insuffisance de l'un d'eux.

\subsubsection{La prosodie :}

La prosodie se définit comme : " L'étude des phénomènes de l'accentuation et de l'intonation( variation de hauteur, de durée et d'intensité) permettant de véhiculer de l'information liée au sens telle que la mise en relief, mais aussil'assertion,l'interrogation, l'injonction, l'exclamation.» (Guzunjhajt, 2010).

La prosodie c'est aussi le domaine particulier de la phonétique qui s'occupe de décrire les sons du langage au niveau de l'énoncé.

\subsubsection{L'accentuation :}

L'accentuation est un phénomène provenant de l'augmentation de la durée syllabique de l'intensité sonore et de la hauteur mélodique sur certaines syllabes de l'énoncé.

Personne ne peut nier l'importance de la perception de l'accent sur la compréhension car elle permet une entrée rapide et efficace dans le texte et si cet accent est mal placé, la compréhension sera certainement affectée.

L'écoute d'un mot mal accentué par un locuteur étranger, ne lui permettra pas de connaître le mot immédiatement, il aura besoin d'un temps supplémentaire et s'il n'arrive pas encore, il devra faire appel à d'autres stratégies.

\subsubsection{L'intonation :}

L'intonation est définit alors comme une structuration mélodique des énoncés en groupe rythmique qui a la particularité de se terminer par une accentuation suivie d'une courte pause. Barry ajoute que les suprasegmentaux

\section{4}




\section{Number 57 , Part 2 , January, 2015}

(intonations, variations mélodiques) permettent aux auditeurs de connaître les voix et de retirer un message précis.

Dans l'exemple ci-dessous nous montrons l'importance de l'intonation pour la compréhension d'un message en déterminant le type de phrases utilisées : affirmatif ou interrogatif. - Vous parler français. - Elle ne comprend pas.

1.3 Les stratégies et les approches utilisées pour améliorer la compréhension orale 1.3.1 Les stratégies de la compréhension orale

Avant d'étudier les différentes catégories des stratégies de la compréhension orale, il est nécessaire de définir le terme de stratégies. Selon Vandergrift, la stratégie « Fait référence aux démarches entreprises par un apprenant pour faciliter sa compréhension, son apprentissage et sa rétention de la langue cible. » (Vandergrift, 2007 : 66)

L'observation de stratégies mises en oeuvre, par les bons apprenants en langue étrangère a donné lieu à de nombreux travaux parmi lesquels citons ceux d'O'Malley et Chamot (1990) qui ont cerné un bon nombre de stratégies d'apprentissage, réparties selon trois catégories : les stratégies métacognitives, les stratégies cognitives et les stratégies socio-affectives.

Les stratégies métacognitives ; qui impliques une réflexion sur l'apprentissage en général et permettent de le mieux planifier et diriger en évaluant ses progrès .L'apprenant qui fait le point sur ce qu'il vient d'apprendre par rapport à son projet met en oeuvre une stratégie métacognitive.

Les stratégies cognitives, quand à elles, sous -entendent une interaction entre le sujet et le matériel d'apprentissage. Ainsi faire des inférences, utiliser ses connaissances

\section{5}

Journal of Arabic Studies in Education \& Psychology (ASEP) 


\section{Number 57 , Part 2 , January, 2015}

textuelles, sont des stratégies liées à l'activité de compréhension orale.

Les stratégies socio-affectives sont mises en oeuvre durant les interactions avec une autre personne pour aider l'apprentissage.

\subsection{2 les méthodes et les approches de la compréhension orale}

Xiaohong (2005) signale que Parmi les méthodes ou les approches les plus célèbres qui accordent une importance à la compréhension orale et ses compétences de ce qui suit :

\subsubsection{La méthode audio-orale}

L'apprentissage d'une langue, alors, devient un processus mécanique permettant à l'apprenant l'acquisition d'un ensemble de structures linguistiques basées sur des exercices structuraux.

Pour cela, l'enseignant fournit des modèles non situationnels d'imitation et de manipulation dont l'objectif n'est autre que la réutilisation des structures apprises.

Le but de la MAO était de parvenir à communiquer et à s'exprimer en langue étrangère, raison pour laquelle on visait les quatre habiletés (compréhension orale et écrite, expression orale et écrite).Cependant, on continuait à accorder la priorité à l'oral suivi de l'écrit après, dans chaque leçon.

\subsubsection{La méthode SGAV}

Guberina présente la langue tel un instrument de communication dont l'apprentissage doit porter sur la compréhension du sens global de la structure, les éléments « audio » et « visuel » facilitant cet apprentissage.

Selon la méthode SGAV, l'apprenant n'a aucun contrôle sur le développement ou sur le contenu du cours, mais, il est actifpuisqu'ildoitcontinuellementécouter,répéter,comprendr e,mémoriseretparler librement. Pour l'enseignant, il est tenu d'animer la leçon par des questions, guidé l'apprenant à y

\section{6}


répondre en stimulant l'activité des apprenants fournies avant.

\subsection{2. $3 \mathrm{~L}$ 'approche communicative}

Selon l'approche communicative, les quatre habiletés peuvent être développées puisque tout dépend des besoins langagiers des apprenants. La langue est vue avant tout comme un instrument de communication et surtout d'interaction sociale. Dans cette perspective, savoir communiquer signifierait préparer l'apprenant aux échanges avec des locuteurs natifs.

Les aspects spécifiquement L'approche communicative présente, au moins pour la compréhension orale, diverses formes linguistiques destinées à transmettre un même message. On prend en compte le niveau du discours et on distingue entre cohésion (les relations existant entre deux énoncés) et cohérence (les relations établies entre des énoncés et la situation extralinguistique). On utilise en classe de préférence la langue étrangère, mais il est possible d'utiliser la langue maternelle et la traduction. En ce qui concerne l'erreur, elle est considérée comme inévitableère ne permettent pas de communiquer efficacement, pour cela l'apprenant doit connaître les règles d'emploi.

L'approche communicative présente, au moins pour la compréhension orale, diverses formes linguistiques destinées à transmettre un même message. On prend en compte le niveau du discours et on distingue entre cohésion (les relations existant entre deux énoncés) et cohérence (les relations établies entre des énoncés et la situation extralinguistique).On utilise en classe de préférence la langue étrangère, mais il est possible d'utiliser la langue maternelle et la traduction. En ce qui concerne l'erreur, elle est considérée comme inévitable.

\subsubsection{L'approche axée sur la compréhension :}

La perspective d'une approche axée sur la compréhension dicte que l'apprentissage d'une langue se fait à travers un

\section{7}




\section{Number 57 , Part 2 , January, 2015}

modèle didactique où l'on envisage les habiletés réceptives comme préalables à la production. Toutefois, l'enseignement d'une langue consiste à préparer l'apprenant à comprendre des textes oraux ou écrits. Aussi, faut-il préciser que cette approche accorde une attention toute spéciale à l'individu qui joue un rôle assez important dans son apprentissage.

Cornaire et Germain déclarent que: « Le bon apprenant est celui qui sait mettre en œuvre des stratégies pour gérer efficacement son apprentissage, tout en essayant de résoudre certaines difficultés inhérentes à des tâches pédagogiques. » (Cornaire et Germain, 1998 : 24).

1.3.2. 5 les TIC (technologies de l'information et de la communication):

Les technologies de l'information et de la communication (TIC) envahissent les pratiques sociales et se développent dans l'éducation. Par ailleurs, l'évolution des sociétés vers une augmentation considérable de l'information et des savoirs rend l'intégration des TIC dans l'enseignement Apprentissage un enjeu qui permet une ouverture sur le monde et qui nécessite diverses études, pédagogiques et didactiques, pour une meilleure utilisation. Utiliser les TIC (technologies de l'information et de la communication) en classe de français langue étrangère signifie enseigner de façon différente par rapport à la classe traditionnelle. (Abdelaziz, 2013 : 9)

Avec le multimédia on passe de technologies qui offraient la possibilité de développer en priorité une seule compétence, orale ou écrite, à des technologies qui rapprochent plus de la multicanalité de la communication réelle. Il permet, de plus, un enseignement-apprentissage du FLE non seulement d'un point de vue linguistique mais également dans les usages culturels de la langue cible. Le multimédia, enfin et surtout, transforme profondément les rapports entre l'enseignant, les apprenants et le savoir. De

\section{8}




\section{Number 57 , Part 2, January, 2015}

dispensateur du savoir il devient guide vers le savoir. (Holtzer, $2007: 73$ )

Les histoires numériques ou les documents audio visuels et motifs sont une des nouvelles applications de TICE en général et de Multimédia surtout. Elles permettent aux apprenants de se familiariser avec divers accents, élément nécessaire au développement de la compréhension. De plus, les documents choisis peuvent présenter divers registres propres à une langue authentique. Grâce à des indices favorisant la compréhension et une contextualisation de la langue dans un cadre précis, la vidéo fournit un support visuel qui facilite l'acquisition du vocabulaire, point d'ancrage de la compréhension. (DELVOTTE et POTHIER, $2004: 52$ )

La construction de la compréhension orale des apprenants face à un document audiovisuel s'effectue la plupart du temps selon les étapes suivantes: dans un premier temps on focalise leur attention sur le visuel en regardant, plusieurs fois, le document sans le son afin qu'ils imaginent ensuite le contenu thématique, les dialogues, à l'aide des indices visuels tels que l'apparence des personnages, leurs faits et gestes, les lieux dans lesquels ils évoluent, etc. Vandergrift, L. $2007: 72$ )

\section{II- La deuxième partie : les histoires numériques II.1 La définition des histoires numériques}

L'utilisation de la technologie est l'un des facteurs les plus importants pour l'enseignement efficace de la langue. La tape, l'appareil enregistreur, le vidéo, la télévision, l'ordinateur, les applications mobiles et l'Internet ont été utilisés dans la technologie soutenue de l'enseignement des langues. L'ordinateur, la téléphone, le mobile, dont l'utilisation a augmenté, font partie indispensable de l'enseignement des langues. (Qteefan, 2012: 10)

Donc d'après (Weis, Benmayor, O.Leary et Eynon 2002; Chung 2006) l'attention à propos du concept de multimédia

\section{9}




\section{Number 57 , Part 2 , January, 2015}

est venue à la suite d'une transition significative dans la pensée pédagogique d'un ensemble de situations éducatives collectives aux situations individuelles. Aussi l'évolution du rôle de l'enseignant, qui est obligé d'utiliser les appareils, les outils et les ressources éducatives de la conception moderne et l'utiliser pour accroître l'efficacité des situations éducatives. On retrouve ici les nouvelles technologies numériques et le multimédia transforment rapidement la façon dont nous enseignons et communiquons à la fois soit dans et hors de la salle de classe et surtout l'environnement technologique pour l'histoire numérique qui se progresse rapidement après le début du XXIe siècle.

L'histoire numérique est l'une des modernes technologies que l'enseignant peut l'utiliser dans la classe comme un changement dans le domaine de l'éducation, Surtout avec les enfants pour de se concentrer sur les opérations de communication à travers les systèmes modernes tels que les logiciels de l'information fournie aux étudiants par les programmes d'ordinateur intégrés graphiquement avec des grandes couleurs, les mouvements et les effets acoustique. (Lasica, 2002 : 23)

En ce sens, l'histoire numérique est un moyen utile pour combiner la narration personnelle avec l'utilisation de la technologie dans les classes. Les histoires numériques sont des projets de la multimédia employant les photos, le vidéo, l'audition, et la musique. Le digital stortelling (DST) prend l'ancien art de la narration orale et s'engage autrement dans une palette d'outils techniques pour tisser les histoires personnelles en utilisant les images, les graphiques et la musique, avec la propre voix de l'auteur. (Skouge et Rao, 2009: 13)

Pour Robin (2008), l'histoire numérique en milieu scolaire est un processus de création des courtes histoires qui permettent aux étudiants et aux enseignants d'améliorer leur collecte d'information et les compétences de résolution

\section{0}




\section{Number 57 , Part 2, January, 2015}

de problèmes et de faciliter la capacité de travailler en équipe collaborative.

D'après Ohler (2008), l'histoire numérique est considérée comme un processus créatif dans lequel une histoire traditionnelle est combinée à la technologie numérique personnel, tel qu'un ordinateur, une caméra vidéo et un enregistreur de sons.

Alors, L'histoire numérique se reflète une amalgame entre l'ancienne narration personnelle et les éléments de la multimédia pour dépasser les obstacles du temps, du lieu et pour aussi faciliter l'imagination visuelle chez les apprenants.

D'ailleurs SKouge et Rao voient que l'histoire numérique est un processus de création d'un court métrage qui combine le script écrit ou le texte original de l'histoire avec les diverses composantes de la multimédia comme les images, les vidéos, la musique, la narration et souvent un commentaire accompagnant l'histoire par la voix du producteur de l'histoire. (SKouge et Rao, 2009 : 54)

D'autre part, Yuksel décrit l'histoire numérique comme un processus créatif dans lequel une histoire traditionnelle est combinée à la technologie numérique personnel tel qu'un ordinateur, une caméra vidéo et un enregistreur de sons. (Yuksel, $2011: 20$ )

De plus, De Natal explique que l'histoire numérique est un court clip (presque 3 ou 4 minutes) qui consiste une série d'images fixes combinée avec le texte oral et ou écrit en ajoutant parfois une musique pour inciter l'émotion ou les autres sentiments chez les apprenants. (De Natal, 2008 : 179)

D'ici, l'histoire numérique peut réaliser l'interactivité chez les apprenants comme une de ses caractéristiques importantes qui se distingue la nouvelle technologie 


\section{Number 57 , Part 2 , January, 2015}

numérique en comparant de l'ancien médias comme ( la cassette, la disque, la magnétophone......)

Dans le cadre de ce qui précède, on peut définir l'histoire numérique comme une nouvelle création à la narration qui fonctionne les multimédias comme la photo, les cartes, les textes, la musique, la voix, le vidéo et les commentaires pour exposer une conte soit réaliste ou imaginaire en réalisant des objectifs cibles.

\section{II.2 Les avantages des histoires numériques}

De nombreux chercheurs des langues (Barrett 2005, Robin 2006, Gregori 2008, Rodriguez 2009, McNeil 2010) ont depuis longtemps mis en évidence l'importance de l'utilisation de la narration numérique dans l'enseignement et l'apprentissage soit dans l'environnement éducationnel en général 1 et soit dans le domaine de l'apprentissage de langue surtout.

L'utilisation du multimédia est considérée comme supports pédagogiques qui peuvent faciliter l'apprentissage et l'enseignement et développer les compétences de la langue étrangère chez les apprenants dans la salle de classe.

Dans le même sens, Tsou et al. (2006) trouvent que l'intégration de l'histoire numérique dans le curriculum de langue est une créative technique pour améliorer le niveau d'apprentissage des compétences de la langue comme la lecture, l'écriture, la locution et l'audition.

Selon Hall, l'histoire numérique peut contribuer à réaliser les effets suivants dans la classe scolaire comme le développement de la créativité et de l'innovation, l'encouragement de la communication et de la coopération, la pratique des compétences de la pensée critique, l'entrainement à la résolution de problèmes et la prise de décision et l'existence de l'apprentissage visuel et auditif efficace). (Hall, 2011:99) 


\section{Number 57 , Part 2 , January, 2015}

L'environnement de l'histoire numérique présente à l'enseignant et aux apprenants des nouvelles situations d'apprentissage non habituées qui peuvent être créer l'esprit de la collaboration et le climat de la pensée afin d'achever l'apprentissage amusant et efficace.

A cet égard, (Frazel, 2010, 10) énumère les avantage des histoires numériques comme suit :

- Motiver les élèves à apprendre le contenu des programmes d'étude.

- Répondre aux besoins de divers groupes d'élèves en prenant de compte les différences individuelles.

- Soutenir l'enseignement en équipe et l'apprentissage dans le programme scolaire.

- Présenter des nouvelles technologies variées pour attirer l'attention des élèves.

- Permettre de rendre parfois l'apprentissage individualisé au sein de l'activité collective de la classe.

De son coté, Gils (2005) voit que les histoires numériques peuvent contribuer à présenter des nouvelles didactiques differciées des méthodes traditionnelles; personnaliser l'expérience d'apprentissage; créer des situations de la vie réelle par un moyens facile et moins cher ; et améliorer la participationdesapprenantsdansle processus d'apprentissage.

Enfin, on peut résumer les avantages de l'histoire numériques dans les points suivants :

- Fournir aux élèves des situations d'apprentissage plus interactifs.

- Améliorer le niveau de la compréhension chez les élèves.

- Obtenir des expériences éducatives plus près de la réalité.

- Favoriser la possibilité de l'apprentissage individualisée ou collective.

- Transmettre les informations variées dans les plusieurs domaines d'étude.

- Encourager l'enseignant à utiliser la technologie moderne dans la salle de classe.

\section{3}




\section{Number 57 , Part 2 , January, 2015}

- Développer l'imagination visuelle et la conscience du lieu chez les apprenants.

- Permettre à répéter les informations et les idées pour que les élèves mémorisent bien.

- Présenter un amalgame entre la philosophie de l'histoire traditionnelle et la narration numérique.

- Renforcer les compétences d'expression et de réception à travers la narration, la chanson, le dialogue, le commentaire et la prononciation.

\section{II.3 Les types des histoires numériques}

La narration numérique peut également prendre différents types de messages, tels que des histoires de survie, de mémorisation, d'aventure et d'accomplissement.

Hotman et al (2010 : 31- 32) classifient l'histoire numérique selon la méthode de préparation comme suit :

- Histoires de photos : les histoires de photos sont des combinaisons d'images fixes et de textes. C'est la meilleure façon de savoir les principes de base de la production vidéo. Les enseignantes doivent savoir comment prendre des photos et comment faire une présentation "PowerPoint" dans lequel les photos seront réunies avec le texte. c'est facile de faire une histoire de photo.

- Mots de vidéo: les mots vidéo sont un groupe de mots ou des phrases et des images pour réaliser un film ou une présentation courte et simple, l'enseignant peut prendre quelques photos et les mettre dans les classes, puis demander aux enfants de faire correspondre les images avec les mots. Si les enfants sont d'accord sur les meilleurs sujets, l'enseignant préparera une vidéo. Celleci peut être réalisée sous la forme d'un puzzle, quizz ou un dictionnaire qui peut être utile pour les enfants.

- Présentation : Le processus de fabrication de l'histoire-le plus commun est une présentation PowerPoint. La présentation est une combinaison d'un texte et des images pour présenter un sujet. On peut résumer les idées les

\section{4}




\section{Number 57 , Part 2, January, 2015}

plus importantes et les présenter dans un certain ordre. Les Présentations groupent à la fois la langue et la multimédia. Les élèves doivent être en mesure de rechercher des informations nécessaires à leur présentation et de décider les faits importants et les pas importants.

- Stage (staging) mise en scène: le Staging est une sorte de présentation mais les enseignantes ne sont pas axées sur des faits, mais ils doivent trouver un moyen comment effectuer leurs sentiments, leurs actions, leurs incidents et leurs énonciations. Les élèves doivent présenter les choses qui sont familières. La performance est réalisée dans l'environnement dont ils savent très bien et où ils se sentent en sécurité.

- Video clips: Dans le vidéo clip, nous mettons des images des mots, des conversations enregistrées ou des narrations et de la musique pour faire un complexe significatif qui couvre tous les types des histoires numériques mentionnées ci-dessus. Un clip vidéo parle d'un certain sujet qui est familier à l'auteur et son point de vue personnel reflète ses sentiments et ses émotions.

Yuksel (2011 : 21) voit que les histoires numériques ont trois genres principaux comme suit :

- Histoires personnelles (narration personnelle) : parler du caractère de la même personne pour exposer les événements, les idées et les concepts.

- Histoires Documentaire (historique) : Parler des événements et des faits historiques pour reconnaître les personnages historiques et enrichir les informations des élèves.

- Histoires éducationnelles : pour donner aux élèves des nouvelles expériences éducatives.

Le chercheur adopte trois catégories principales des histoires numériques comme suit :

- Histoires personnelles : Dans ce type, la personne raconte ses propres expériences, ses souvenirs et ses évènements. 


\section{Number 57 , Part 2 , January, 2015}

- Histoires documentaires : Dans ce type, on présente des événements historiques et descriptifs.

- Histoires éducatives ou instructives : Dans ce type, on enseigne des matières spécifiques à un public

\section{II.4 Les éléments de l'histoire numérique}

Des nombreux chercheurs ((Lambert, 2003 ), (Bull \& Kajder, 2004 ), (Banaszewski, 2005), (Robin, 2008 ), (De Natal, 2008 ), (Shelley, 2011) s'accordent que les histoires numériques se fondent sur quelques éléments de la narration comme suit :

\section{Point de vue:}

Le point principal de l'histoire et la perspective de l'auteur. Il faut aussi examiner la raison de l'histoire. Et l'intérêt de cette histoire pour ce groupe de personnes.

\section{Une question dramatique:}

C'est la question que les élèves vont répondre à la fin de l'histoire, et c'est importante pour susciter la curiosité des élèves et les impliquées dans l'histoire et sera donné une explication à la fin de l'histoire.

\section{Contenu émotionnel}

Les émotions importantes que l'histoire numérique se suscite chez les élèves At - il vous rendre triste, heureux, excité, etc.? Comment pouvez-vous améliorer le contenu émotionnel à travers l'imagination visuelle?

\section{Le don de voix :}

Une façon de personnaliser l'histoire pour aider les élèves à comprendre le contexte. Personnalise l'histoire, aide avec les apprenants de langue anglaise (ELL) d'instructions, scripts, etc. On peut aussi utiliser sa voix pour transmettre le message de sorte que soit alignée avec l'imagerie.

\section{La puissance d'une Soundtrack :}

Le pouvoir du Sound trac soutient l'histoire avec la musique appropriée. Musiques de mettre dans l'ambiance de nos jours, changer la façon dont nous percevons l'information visuelle en continuant dans les yeux, et établir un rythme de notre démarche. Voici trois extraits sonores

\section{6}




\section{Number 57 , Part 2 , January, 2015}

très différents qui peuvent démontrer à vos élèves, les bruits d'impact peuvent avoir une ambiance chaleureuse.

\section{6. Économie}

On doit éviter surcharger le spectateur avec un usage excessif de moyens visuels et / ou auditif; utiliser le contenu pour présenter l'histoire sans beaucoup d'informations.

Si l'écrivain et l'émetteur font un bon travail, ils prennent tout ce qui est nécessaire pour garder visuellement l'histoire riche se déplaçant à travers l'histoire par un minimum de dialogue et un ensemble de scènes nécessaires pour nous permettre à reconnaitre l'histoire plus rapide.

\section{Accélérer}

Le rythme d'une histoire détermine précisément l'intérêt acquis à part d'un auditeur. Un rythme lent de l'histoire peut être favorisé la contemplation, le romantisme, la relaxation, ou les plaisirs simples. Donc le rythme de l'histoire soit au sujet de la raconte ou soit au sujet de la multimédia.

D'autre part, Shelley (2011 : 11) confirme qu'il y a des facteurs qui se contrôlent la réussite de la préparation de l'histoire numérique. Ces facteurs comme suit :

\section{La bande dessinée}

L'utilisation des photos caricatures aide à transférer clairement les sentiments des personnages et minimiser le dialogue qui peut être exprimé ces images.

\section{Une chronologie interactive}

Lorsque l'histoire raconte un point de vue, il faut coordonner le calendrier de la chaîne des événements et leurs conséquences de manière interactive pour attirer l'attention des apprenants.

\section{Les médias sociaux}

On peut fonctionner les histoires courtes comme source d'inspiration pour discuter un sujet éducatif. L'apprentissage électronique peut aussi encourager les apprenants à partager leurs histoires liées au thème de la leçon pour concevoir les points importants et les idées principales.

\section{7}




\section{Number 57 , Part 2 , January, 2015}

\section{La voix du narrateur}

La bonne voix du narrateur de l'histoire numérique est un facteur qui rend les élèves de vivre directement et émotionnellement au message transmis par l'histoire numérique. Les chercheurs et les experts sont d'accord que le narrateur du texte littéraire peut défavoriser l'apprenant plus que l'aider.

\section{Le vidéo}

Il possible de utiliser les vidéos dans l'histoire pour donner aux élèves le contact humain. C'est pourquoi, on doit favoriser l'accord entre le narrateur et les slots de vidéo dans la préparation des histoires numériques.

Donc, on peut dire que s'on favorise quelques éléments, on va préparer une histoire numérique réussie. Ces élément peut comporter le sujet ou le point de vue ou la valeur présentée; la qualité du scénario, la voix du narrateur, les éléments de la multimédia et la coordination convenable entre ces éléments.

\section{II.5 Les étapes de la préparation de l'histoire numérique}

Beaucoup de recherches et d'études comme (Bull et Kajder 2004) ; (Chung 2008); (Erin et Miller 2009); (Hussein Abdul basset 2010) ; (Merwad, .2013) limitent les étapes de la préparation de l'histoire numérique comme suit:

- Les objectifs de l'histoire numériques.

- Le contenu de l'histoire numérique.

- Le multimédia utilisés dans l'histoire.

- La Production de l'histoire numérique.

- Le mouvement nécessaire de l'histoire.

Déterminer les objectifs de l'histoire numériques

- Identifier l'objectif principal de l'histoire à travers la valeur cible.

- Limiter le nom convenable de l'histoire à la lumière de l'idée présentée.

- Choisir les vocabulaires qui se reflètent les objectifs et les idées de l'histoire.

\section{8}




\section{Le contenu de l'histoire numérique}

- Écrire le scénario de l'histoire et préparer le story-board en les reliant. Le scénario doit être court et déterminé. Le scénario doit contenir un début, une idée principale, l'intrigue, les personnages, le style adéquat et le point de vue de l'auteur et les questions qui nécessitent la pensée de l'élève.

- Donne à l'élève un point de référence et un point de départ.

- Lieu (maison, école, terrain de jeux, etc).

- Temps (aujourd'hui, parfois dans le passé, dans le futur).

- Météo (C'était une nuit sombre et orageuse ...).

- Présenter les séquences de l'histoire (le début, le milieu et la fin). Dans la parcelle, on élabore ce qui suit :

- Une introduction ou le début de l'histoire où on rencontre les personnages.

- Rising Action, c'est-à-dire que - quelque chose qui se passe tout au long du chemin provoquant une sorte de conflit, la question essentielle (le garçon va gagner la fille, vaincre le dragon, etc)

- Climax - c'est la partie la plus intense de l'histoire et, en fait, le point central de l'histoire. Le public est accroché et on nous demande si les personnages vont résoudre le conflit.

- Chute d'action - le public commence à voir (si) et (comment) le conflit a été / n'a pas été résolue.

- Dénouement - le résultat final de tout ou la fin de l'histoire.

- Conflits : le conflit ou la question essentielle est la raison de l'intrigue. C'est ce qui conduit les personnages et le public à travers l'histoire et nous conduit finalement à la fin de l'histoire.

- Personnages : Un personnage peut être vous-même, ou un protagoniste (bon gars), et / ou antagoniste (méchant).

\section{9}




\section{Number 57 , Part 2 , January, 2015}

- Scripting : Les élèves tournent autour d'un script de 200300 mots qui deviendra l'audio pour leurs histoires. Les pairs et l'instructeur peuvent poser des questions et formuler de feedback sur le script.

- Storyboarding : les élèves montrent comment les mots dans leurs script synchroniser avec les images qu'ils ont l'intention d'utiliser dans leurs histoires. C'est une nouvelle occasion pour le feedback .Le scripte doit contenir une question dramatique et émotionnelle. Cette question attire l'attention des élèves à la fin de l'histoire ou on peut trouver la solution.

\section{Le multimédia utilisés dans l'histoire}

L'enseignant collecte un nombre convenable des textes, des images, des graphiques, du background musical, du vidéo, et les programmes d'ordinateurs. les multimédia s'intègrent pour réaliser l'objectif de l'histoire.

Les enseignantes ajoutent des transitions, des titres et des crédits. Il est important pour cette dernière à venir, que les transitions peuvent modifier le calendrier d'une pièce. Les élèves exportent leurs projets et les graver sur CD ou DVD au moins une copie de l'élève et l'autre pour l'enseignant.

Il est important de planifier une examination finale afin que les étudiants puissent présenter et discuter leurs travaux. Le processus de créer l'histoire ne nécessite aucun logiciel spécialisé. Nous pouvons utiliser des programmes tels iphone, iMovie, power point ou Windows Movie Maker et certains de ces programmes sont aussi disponibles sur nos ordinateurs. Tous les projets qui sont achevés peuvent être partagées et réparties sur CD, DVD or web sites.

\section{Le mouvement dans l'histoire numérique.}

La désignation du mouvement de la multimédia a pour but d'attirer l'attention des élèves et de transmettre les sentiments et les émotions des scènes. Il ya une synchronisation appropriée entre la multimédia utilisée, y compris le rythme rapide dans le récit des événements, dans

\section{0}




\section{Number 57 , Part 2 , January, 2015}

les scènes qui nécessitent la passion du spectateur, et le rythme est lent dans le récit des événements qui nécessitent une réflexion. Le ton varie avec la voix du narrateur de l'histoire selon les événements historiques et les situations qui exigent). Avant de créer l'histoire numérique, il faut prendre en compte certaines d'aspects comme l'âge des élèves, et les buts qu'on veut achever, les compétences de la langue lesquelles on désire développer ou améliorer.

\section{II.6 Les applications pédagogiques des histoires}

\section{numériques}

L'histoire numérique est considérée comme un moyen, une stratégie et une méthode qui encouragent les enseignants et les élèves à pratiquer la créativité de l'apprentissage. La narration numérique favorise plusieurs potentialités comme la voix, les textes, les images, l'audio et le vidéo. Ces possibilités attirent l'attention des apprenants et favorisent un environnement d'apprentissage créatif qui incite la pensée et l'amusement.

Frazel (2010:10-11) énumère les applications des histoires numériques dans la salle de classe comme suit :

- Développer la créativité et l'innovation : l'exploitation des connaissances existantes pour générer des nouvelles idées, et créer des œuvres originales qui reflètent une personne ou un groupe.

- Développer le contact et la coopération : l'interaction avec les pairs et les experts en utilisant des environnements numériques.

- Développer la pensée critique, la résolution de problèmes et la prise de décision : la planification et la gestion des activités visant à trouver des solutions ou à réaliser un projet.

- Développer les opérations et les concepts de la technologie : employer les applications de manière efficace et productive; transmettre des nouvelles technologies pour l'éducation. 


\section{Number 57 , Part 2 , January, 2015}

A son coté, Smilack confirme que les histoires numériques accompagnées les curricula des langues peuvent renforcer les compétences réceptives et productives des élèves, améliorer les performances de l'écriture grâce à des introductions intéressantes, un apprentissage typique, des structures claires de phrases, un processus d'écriture qui comporte le brainstorming, les projets multiples et la participation. (Smilack, $2007: 4$ )

Bull et Kajder (2004) ont également employé l'histoire numérique dans le domaine de la langue en basant sur le concept de «tout le monde a une histoire à raconter qui vise à pratiquer l'expression personnelle.

Les usages éducatifs de l'histoire numérique permettent aux élèves d'exploiter le don de leur propre voix et l'expression de leurs personnelles idées pour faciliter leur compréhension. L'utilisation de leur propre voix unique donne aux élèves d'une manière personnelle et significative. (Lambert, 2003 : 22)

Donc, Les histoires numériques ont des effets importants à plusieurs niveaux scolaires où la narration numérique aide les élèves à apprendre bien, à effectuer des recherches, à acquérir des expériences plus proches de la réalité, à pratiquer les outils de communication et de création numérique ; à organiser ces idées pour les présenter d'une manière significative et créative qui se reflète l'apprentissage amusant et innovant.

\section{Conclusion :}

On peut conclure que les histoires numériques sont considérés une nouvelle attitude du multimédia moderne pour renforcer, la mémorisation, la compréhension, la communication et l'expression grâce à des nouveaux environnements d'apprentissage créatifs. Ces nouvelles technologies aident les enseignants et les élèves à évoluer ensemble en achevant le développement professionnel à

\section{2}




\section{Number 57 , Part 2 , January, 2015}

travers de la transmission de méthodes traditionnelles à des pratiques pédagogiques innovantes dans un environnement d'apprentissage du multimédia qui encourage la pensée, la participation, la recherche, la création. La narration numérique supporte les situations d'expression et de compréhension orale par les programmes d'ordinateurs intégrés graphiquement avec des différentes couleurs, les mouvements et les effets acoustiques.

\section{2- L'Etude Expérimentale}

Etant donné que notre étude vise à déterminer l'effet des histoires numériques sur le développement des compétences de la compréhension orale en Français chez les élèves du cycle primaire aux écoles des langues. Le chercheur a élaboré une liste des compétences de la compréhension orale et un test de la compréhension orale visant à évaluer ces compétences chez les élèves du cycle primaire.

\subsubsection{Les objectifs de la liste :}

Cette liste vise à déterminer les compétences de la compréhension orale nécessaires aux élèves du cycle primaire dans les écoles des langues.

\subsubsection{Les sources de la liste :}

Pour préparer cette liste, le chercheur a recours aux sources suivantes :

- les études et les recherches antérieures qui ont abordé la compréhension orale et ses compétences.

- Les analyses théoriques contenues dans les références suivantes :

1. Cornaire, C. \&Germain, C. (1998). La compréhension orale, Paris, Clé International.

2. FERROUKHI, K. (2009). " La compréhension orale et les stratégies d'écoute des élèves apprenant le français en 2e année moyenne en Algérie". in Synergies, pp. 273280.

3. MASUY, F. \& GENEVIEVE, B.(2010). Stratégies pour une écoute efficace, Belgique, Université catholique de Louvain-la-Neuve. 
4. MAURER, B.(2001). Une didactique de l'oral. Du primaire au lycée, Paris, Lacoste.

5. Michaud, E.(2010)." Entraîner, évaluer la compréhension orale". Académie de Versailles, octobre.

2.1.3 La description de la liste des compétences de la compréhension orale :

La liste des compétences de la compréhension orale en français a comporté 15 compétences nécessaires aux élèves du cycle primaire dans les écoles des langues. Devant chaque compétence se trouve le degré d'importance (très important - important - peu important).

\subsection{Elaboration du test de compréhension orale :}

Notre recherche a pour but de déterminer l'effet des histoires numériques sur le développement des compétences de la compréhension orale chez les élèves du cycle primaire dans les écoles des langues. Le chercheur a poursuivi les étapes suivantes :

1. Identification des objectifs du test.

2. Détermination des sources du test.

3. Description du test.

4. Passation du test :

- Choix de l'échantillon.

- Durée du test.

- Fidélité du test.

- validité du test.

- notations du test.

\subsubsection{Les objectifs du test :}

2.2.1.1 objectif général :

Ce test vise à évaluer l'effet des histoires numériques sur le développement des compétences de la compréhension orale chez les élèves du cycle primaire dans les écoles des langues.

\subsubsection{2 objectif spécifique :}

Notre test de compréhension orale a pour but de mesurer chez les élèves du cycle primaire dans les écoles des langues les compétences suivantes :

\section{4}




\section{Number 57 , Part 2 , January, 2015}

- Distinguer correctement les mots ayant des mêmes sonores.

- Discriminer correctement les différents sons.

- Chercher des informations précises en écoutant attentivement le texte.

- Associer des illustrations à des messages oraux.

\subsubsection{Détermination des sources du test :}

Pour préparer le test, le chercheur a révisé de ce qui suit :

- les études et les recherches antérieures qui ont abordé la compréhension orale.

- Les ouvrages comme les livres, les magazines et les articles qui ont évalué la compréhension orale.

\subsubsection{Description du test :}

Après avoir limité les objectifs et les sources du test, on a mis les questions de ce test pour évaluer le degré de maitrise des compétences de la compréhension orale chez les élèves du cycle primaire dans les écoles des langues avant et après l'application des histoires numériques.

Ce test comprend quatre questions portant sur les compétences de la compréhension orale choisies. On compte 20 notes pour chaque question. Donc la note totale du test est 80 notes.

Les quatre questions ont comporté les situations suivantes de la compréhension :

- Ecoutez le texte suivant qui explique les ingrédients de la préparation de la pâte à crêpe, puis cochez parmi les ingrédients qui sont citées dans le texte enregistré.

- Ecoutez les dix phrases suivantes, puis choisissez (oui) ou (non) si la phrase que vous lisez correspond à la phrase que vous entendez.

- Vous allez entendre pour deux fois l'histoire actuelle, puis trouvez des réponses convenables aux questions cidessous.

- Ecoutez le texte ci-dessous, puis soulignez les mots qui ne sont pas dans le texte. 


\section{Number 57 , Part 2 , January , 2015}

\subsubsection{Passation du test :}

Avant la passation du test, le chercheur définit les points suivants :

\subsubsection{Choix de l'échantillon :}

L'étude expérimentale de notre étude se sert d'un seul groupe parmi Un échantillon d'élèves de la de la cinquième année primaire d'une d'écoles des langues à Damiette.

\subsubsection{Durée du test :}

On a calculé le temps du test en prenant la moyenne du temps entre le premier et le dernier étudiant. La durée de l'application du test fut 80 minutes.

\subsubsection{Fidélité du test :}

Pour calculer la fidélité du test, nous avons fait deux passations à la fin d'Novembre 2014, puis deux semaines après. Nous avons calculé la corrélation entre les deux passations en suivant la formule statistique de spirman (ElBahy, 1997)

Indice de fidélité $=2 \mathrm{R}$

$$
1+\mathrm{R}
$$

Donc, la fidélité du test était 0,74

\subsubsection{4 validité du test :}

Nous avons présenté le test aux membres du jury pour voir si le test est convenable au niveau des élèves de la de la cinquième année primaire des écoles des langues. Le chercheur a pris en considération toutes les remarques proposées par les membres du jury.

Aussi, on a calculé la validité du test à partir de sa fidélité en appliquant la formule suivante (El-Bahy, 1979).

\subsubsection{5 notations du test :}

Nous avons consacré 80 notes à la note totale du test. Ces notes sont distribuées aux quatre questions (20 notes pour chaque question).

\subsection{Elaboration des histoires numériques:}

Afin de préparer les histoires numériques pour développer les compétences de la compréhension orale

\section{6}




\section{Number 57 , Part 2 , January, 2015}

d'élèves de la cinquième année primaire des écoles des langues, nous avons entrepris les étapes suivantes :

- Délimiter les objectifs des histoires numériques.

- Déterminer les sources des histoires numériques.

- Elaborer le contenu des histoires numériques.

2.3.1 les objectifs des histoires numériques

Notre programme basé sur les histoires numériques a pour but de développer les compétences de la compréhension orale chez les d'élèves de la cinquième année primaire des écoles des langues. Ces compétences comportent les suivants:

- Distinguer correctement les mots ayant des mêmes sonores.

- Discriminer correctement les différents sons.

- Chercher des informations précises en écoutant attentivement le texte.

- Associer des illustrations à des messages oraux.

2.3.2 Déterminer les sources des histoires numériques

Pour préparer le programme proposé, le chercheur a eu recours aux sources suivantes :

- Popet, A. (2006). " Le conte au service de l'apprentissage de la langue". Conférence du 5 avril 2006, Cddp Boulogne.

- Chung, S. (2008). "Digital Storytelling in Integrated Art Education. The International Journal of Arts Education'. Vol. (4), No(1), pp $33-50$.

- Alexander, B . (2011). The new digital storytelling Creating Narratives with New Media, Santa Barbara, California , U. S. A.

- Bozdogan, D. (2012)."Content analysis of ELT students digital stories for young learner, Novitas-ROYAL (Research on Youth and Language), Vol.(6) No. (2), pp.126-136.

2.3.3 Elaborer le contenu des histoires numériques

On a élaboré quatre histoires numériques visant à développer les compétences de la compréhension orale chez

\section{7}




\section{Number 57 , Part 2 , January, 2015}

les élèves de la cinquième année à travers un thème proposé à traiter. Ces histoires comportent des supports intéressants comme des bandes dessinées, des images, des clips vidéos, un rétroprojecteur et un $\mathrm{PC}$ avec vidéo projecteur pour convoquer l'imagination, la sensation des élèves ; aussi leur mettre en contact dans la culture française et favoriser également les chances afin de pratiquer les compétences de la langue française.

Les nominations des histoires numériques sont :

- Mon ami Crocodile.

- Un autre Monde.

- Toute la vérité sur le père Noël.

- Zila et le Chevalier.

\subsection{Enseignement des histoires numériques :}

Après avoir appliqué le pré-test, on a enseigné les histoires numériques en deuxième semestre de l'année scolaire 2014 - 2015 à partir 25 mars jusqu'à 22 avril. On a enseigné ces histoires aux élèves de la de la cinquième année primaire d'une d'écoles des langues à Damiette comme un groupe expérimental.

Cette application a duré 4 semaines à moyenne trois rencontres par semaine (à raison quarante cinq minutes pour chaque rencontre).

\subsection{Application du Post - Test :}

A la fin de l'enseignement de nos histoires numériques, nous avons réappliqué le même test de la compréhension orale (pré - post -test) 23 avril 2015 aux sujets de notre groupe expérimental afin de détecter le degré d'amélioration des élèves aux compétences de la compréhension orale. Ainsi nous passons à l'analyse statistique des résultats.

\subsection{Analyse des résultats :}

Pour vérifier notre première hypothèse, notre analyse statistique suit la technique suivante :

La moyenne arithmétique des notes, l'écart type et la valeur de "T" selon l'équation suivante (El-Bahy, 1979)

\section{8}




$$
\begin{aligned}
& \text { 1) } M=\frac{T N}{\text { ห }} \\
& \text { 2) } E=\frac{T^{2}\left(T N^{2}\right)}{\text { ห }} \\
& \text { 2) } T=\frac{T N D}{T N(D)^{2}(T N D)^{2}} \\
& \text { ห- } 1
\end{aligned}
$$

Tableau No.1Les résultats relatifs au pré- post de la compréhension orale

\begin{tabular}{|c|c|c|c|c|c|c|c|c|}
\hline Test & $\mathrm{N}$ & $\mathrm{M}$ & $\mathrm{E}$ & $\mathrm{D}$ & $\mathrm{D}^{2}$ & $\begin{array}{c}\mathrm{T} \\
\text { Calculé }\end{array}$ & $\begin{array}{c}\mathrm{T} \text { au } \\
\text { Tableau }\end{array}$ & $\mathrm{S}$ ou \\
\hline$\frac{\text { Pré-Test }}{\frac{\text { Post }-}{\text { Test }}}$ & 33 & $\frac{10.87}{21 .}$ & $\frac{1.99}{3.83}$ & 10.44 & 108.9 & 13.46 & 2.39 & 0.01 \\
\hline
\end{tabular}

Le tableau ci-dessus indique le total des notes. Le total des carrés des notes, la moyenne arithmétique, l'écart type et la valeur de "T'au pré/post test de la c orale.

Nous remarquons qu'il y a une différence statistiquement significative entre les moyennes des notes du groupe expérimental au pré et post-test des compétences de la compréhension orale en faveur du post-test.

La valeur de "T" calculé (13.46) est plus grande que celle du tableau (2.39) au niveau de $p>01$. Ce qui met en valeur la progression dûe à l'utilisation de nos histoires numériques:

- Les histoires numériques ont développé les compétences de la compréhension orale chez les élèves du cycle primaire grâce à un climat d'encouragement et de motivation qui contribue à exprimer librement et de comprendre soigneusement la langue parlée.

- les techniques des histoires numériques ont favorisé les occasions d'interaction entre les élèves à travers des groupes. Cette interaction leur permet échanger leurs expériences et leurs connaissances en supportant les compétences de la compréhension orale.

\section{9}




\section{Number 57 , Part 2 , January, 2015}

- Les histoires numériques rendent l'enseignement plus amusant et plus efficace. Par conséquent, les attitudes des élèves deviennent plus positives envers la langue française.

- Les histoires numériques sont considérées comme un moyen tangible pour développer les capacités de la pensée critique, de l'imagination et de la concentration chez les élèves en supportant leur efficacité et leurs compétences de la compréhension pendant l'acte de parole.

2.7 Calcul de l'effet du programme proposé basé sur les histoires numériques :

Pour vérifier l'effet de notre programme proposé, nous utilisons la formule suivante (Ghassan youssef 2009) :

Nous calculons le Carré d'Etta $\boldsymbol{\eta}^{2}$ selon l'équation suivante :

$$
\begin{aligned}
& \eta^{2}=\frac{t^{2}}{t^{2}+d d l} \\
& \mathbf{t}^{2}=\text { valeur de } \mathbf{T} \text { calculé. } \\
& \text { ddl }=\text { Degré de Liberté }(\mathbf{N}-\mathbf{1})
\end{aligned}
$$

Le carré d'Etat était 5.1 comme une grande valeur. Ce qui met en relief le rôle effectif de notre programme basé sur les histoires numériques à développer les compétences de la compréhension orale choisies chez les élèves du cycle primaire aux écoles des langues.

\subsection{Recommandations de la recherche :}

A la lueur des résultats de la recherche, nous pourrions recommander de (d') :

- Exploiter les modernes moyens technologiques pour développer les compétences de la langue française dans les différents cycles d'étude.

\section{0}




\section{Number 57 , Part 2 , January, 2015}

- Entrainer des apprenants aux compétences de la compréhension orale dans les différents cycles éducatifs en général et dans le niveau primaire en particulier.

- Evaluer systématiquement et exactement les méthodes, les stratégies et les outils utilisées pour enseigner les compétences de la compréhension orale aux futurs enseignants au niveau de l'enseignement universitaire ou aux apprenants au niveau de l'enseignement avant universitaire.

- Entrainer les enseignants pendant la service à employer les supports technologiques comme les histoires numériques et le portable dans l'enseignement des compétences de la compréhension orale pour rendre le processus d'apprentissage plus efficace et plus interactif avec les apprenants.

- Nécessiter à fonctionner la technologie moderne dans l'enseignement en général et dans l'enseignement du Français surtout pour rendre le climat d'apprentissage plus amusant et plus interactif en réalisant des bons résultats .

- Effectuer un nombre de recherches et d'études qui s'occupent des difficultés d'apprentissage des compétences de la langue française aux différentes disciplines éducatives en général et au niveau universitaire en particulier.

\subsection{Suggestions de la recherche}

A partir des résultats obtenus par notre étude, le chercheur suggère les thèmes suivants en langue française :

- Vérifier l'efficacité des histoires numériques pour developper les competences de l'expression orale chez les débutants de l'apprentissage de la langue française.

- Effectuer une étude pour évaluer les méthodes et les curricula utilisées pour enseigner les competences de la compréhension orale dans les différentes disciplines éducatives.

- Etudier l'influence des histoires numérqiues sur l'acquisition des élèves du cycle primaire des attitudes

\section{1}




\section{Number 57 , Part 2 , January, 2015}

positives et reduire l'anxiété linguistique envers la matière du français et ses différentes compétences.

- Investiguer le rôle des histoires numériques dans le developpement des competences de l'expression écrite chez les élèves du cycle primaire.

- Elaborer un programme proposé basé sur les histoires numériques pour augmenter les vocabulaires en Français chez les élèves du cycle primaire.

\section{BIBLIOGRAPHIE}

- Abdelaziz, B.(2013). " L'usage des TICE en classe de FLE : quel dispositif pour quelles compétences dans l'enseignement secondaire". Thèse de Magistère, Université Mohamed Kheider Biskra.

- Alrabadi, E.(2010)." Quelle méthodologie faut-il adopter pour l'enseignement/apprentissage de l'oral ?".Thèse de doctorat, Université du Yarmouk.

- Ashm- Yetis, V.(2012). " Travailler la Compréhension orale avec des Balados : une Etude avec des Apprenants de FLE". Thèse de Maitrise, Université Anadolu.

- Banaszewski, T.(2005)."Digital Storytelling Finds Its Place in the Classroom". Multimedia Schools, V.9, N.1, PP.32 - 35.

- Barrette, H.(2005). "Researching and Evaluating Digital Storytelling As A Deep Learning Tool". C. Crawford (Eds.) Proceeding of Society for Conference, Chesapeake, VA : AAC, 2006.

- Belair, L.(2012). " Mieux Comprendre à L'oral et à l'Ecrit Pour Mieux Communiquer ". Institut National de Formation Pédagogique, Quebec, Canada.

- Bull, G\& Kajder, S.(2004). "Digital Storytelling in the Language Arts Classroom". Journal of Learning and leading with Technology. V.32, N.4, FEV.

- Carette, E. 2001. "Mieux apprendre à comprendre l'oral en langue étrangère" Journal du Français dans le Monde. Recherches et applications, Paris, CLE International, Janvier, pp.128-132.

- Charle, R \& William, C.(1997). La communication orale, Paris, France

- Chung, S.(2008). "Digital Storytelling in Integrated Art Education". International Journal of Education Arts, V.4, N.1, PP.

- Cornaire, C. \&Germain, C. (1998). La compréhension orale, Paris, Clé International.

- Cornaire, C. \& Raymond, P.(1999). La Production Ecrite, Paris, Clé International. 


\section{Number 57 , Part 2 , January, 2015}

- Defay, J. \& Deltour, S.(2003). Le Français Langue Etrangère et Seconde : Enseignement et Apprentissage, Liège, Margade.

- Delvotte, C. \& Pothier, M.(2004). "La notion de ressources à $l^{\prime h e u r e ~ d u n u m e ́ r i q u e " . ~ N o t i o n s ~ e n ~ q u e s t i o n s, ~} \mathrm{~N}^{\circ} 8$, ENS Editions.

- De Natale, G.(2008). Digital Storytelling Tips and Resources , Simmons College, Boston.

- Embarki, A.(2012). "La chanson comme support didactique pour améliorer la compréhension et l'expression orales". Thèse de Magistère, Université Elhadj Lakhdar Batna.

- Erin, E. \& Miller, M.(2009)."Digital Storytelling as Cultural Historical Activity: Effects on Information Text Comprehension ". The degree of doctor, The University of Miami.

- Ferroukhi, K.(2009). "La Compréhension Orale et les Stratégies d'Ecoute des Elèves Apprenant le Français en 2 ème Année Moyenne en Algérie". Thèse de Doctorat, Université de Blida.

- Frazel, M.(2010)." Digital Storytelling Guide for Educator". International Society for Technology in Education, Oregon, Washington.

- Gaonac'h, D. (1990). Théories d'apprentissage et acquisition d'une langue étrangère. Paris : Hatier, CREDIF.

- Gil, F.(2005)."Potential Applications of Digital Storytelling in Education". The degree of master, Faculty of computer Sciences, University of Twente.

- Ginet, A.(2007). Du laboratoire de Langue à la Salle de cours multimédias, Paris, Nathan.

- Green, A.(2011). "Teaching the Writing Process through Digital Storytelling in Pre-service Education". Dissertation of Doctor, University of Texas at Austin.

- Gregori, C.(2008). "Integrating the Old and the New: Digital Storytelling in the EFL Language Classroom". Journal of Greta, V.16, N.1, PP.29- 35.

- Gremmo, M. \& Holec, H. (1990). " La compréhension orale : un processus et un comportement" Le Français dans le Monde, Février - Mars.

- Guzunjhajt, E.(2010). " La Prosodie " Disponible de (http://www.linguistes.com/phonetique/prosodie.html).

- Hall, T.(2011). "Digital Renaissance: the Creative Potential of Narrative Technology in Education". Journal of Creative Education, V.3, N.1, PP. $96-100$.

- Harmer, J.(2007). The practice of English language teaching, Fourth edition. Harlow, Pearson Longman.

- Holtzer, G.(2007).“Conduites et stratégies dans l'apprentissage d'une langue étrangère à distance, dans Oudart, $\mathrm{P}$ (dir.) 


\section{Number 57 , Part 2 , January, 2015}

"Multimédia, réseaux et formation“, Le Français dans le Monde, $\mathrm{n}^{\circ}$ spécial, Juillet 1997.

- Hotman, A.; Hull, A. \& KaMAL, R.(2010). "Video Production and Language Learning" available from (http: // divisproject.eu/attachments/143_DIVIS-Mini- Guide- English .PDF

- Lafontaine, L.(2001). " Enseigner le français oral au Québec : vivre la variété des usages et des normes". Journal du Français dans le Monde, $\mathrm{N}^{\circ}$ spécial : Oral : variabilité et apprentissages, Paris, CLE International, PP. 175-182.

- Lafontaine, L.\& Prefontaine, e.(2007). " Modèle didactique descriptif de la production orale en classe de français langue première au secondaire". Revue de sciences de l'éducation, Vol.33, No.1, PP. 47 - 66.

- Lambert, J.(2003). "Digital Storytelling Cookbook and Traveling Companion". Available from (www.storycenter.org/cookbook.pdf).

- Lasica, J. (2002). Digital tools easier to grasp. USC Annenberg Online Journalism Review. Retrieved on mai 27, 2014 available from (http://www.ojr.org/ojr/lasica/1034121182.php).

- Masuy, F. \& Geneviève, B.(2010). Stratégies pour une écoute efficace, Belgique, Université catholique de Louvain-la-Neuve.

- MAURER, B.(2001). Une didactique de l'oral : Du primaire au lycée, Paris, Lacoste.

- Michaud, E.(2010)." Entraîner, évaluer la compréhension orale". Académie de Versailles, octobre.

- McNeil, H.(2010). "Digital Storytelling in Higher Education". Journal of computing in Higher Education, V.19, N.1, PP.65 - 79.

- Nonnon, E.(2000). " La parole en classe et l'enseignement de l'oral : champs de référence, problématiques, questions à la formation", en Recherches, 33, Presses de 1'université Charles-de-Gaulle Lille3, 75-90.

- Ohler, J.(2008)."The World of Digital Storytelling". Journal of Educational Leadership, Vol.63, N.4, January, PP.44 - 47.

- Porcher, L.(2005). Le Français Langue Etrangère, Paris, Hachette Education.

- Qteefan, Gh.(2012). "The Effectiveness of Using Educational Computer Games on Developing Palestinian Fifth Graders Achievement in English Language in Gaza Governorate". Master Degree in Education, The Faculty of Education, The Islamic University of Gaza.

- Robin, B. (2006). "The Educational Use of Digital Storytelling". In C.Crawford et al.(Eds.), Proceedings of Society For Information 


\section{Number 57 , Part 2 , January, 2015}

Technology \& Teacher Education International Conference 2006(pp.709-716). Chesapeake, VA:AACE.

- ROBIN, R.(2008)." Digital Storytelling: A Powerful Technology Tool for the 21 St Century Classrooms the College of Education and Human Ecology". Theory into Practice, N.47, PP.220 - 228.

- RODRIGUES, C.(2009). " Aide à l'apprentissage du Vocabulaire Dans un Environnement Hypermedia en Français Langue Etrangère". Thèse de Doctorat, Faculté des sciences, Université Clermont II.

- Rost, M.(2002). Teaching and researching listening, London: Longman.

- Séverine, S.(2000)."Un oral, des « oraux », et autres voies orales". Presse de l'université Charles-de-Gaulle, Lille3, 29-59.

- Shelley, A.(2011). "Storytelling in E-Learning: the Why an How Elearn Magazine". Educational and Technology in Perspective. , N.27, April.

- Skouge, J.\& Rao, K. (2009)."Digital Storytelling in Teacher Education: Creating Transformations through Narrative". Faculty of Education, University of Hawaii.

- Smilack, J.(2007). " Steeped In History, Light on Technology: A Genre Analysis of Digital Storytelling Guidebooks ". The degree of Master, Faculty of the Graduate School, University of Colorado.

- Soussi, A. \& Martin, D.(1999)."Comprendre l'oral n'est pas si facile". Neuchâtel : IRDP, pp.374-390.

- Taghezout, M.(2009). "L'activité Ludique dans L'apprentissage des Mots en Français Langue Etrangère". Mémoire de magistère, Faculté des Lettres, des Langues et des Arts, Université D'Oran.

- Tsou, W.; Wang, W. \& Tzeng, Y.(2006). "Applying a Multimedia Storytelling Website in Foreign Learning". Journal of computer and Education, N.47, PP.17 - 28.

- Vandergrift, L. 2007. " L'enseignement de la compréhension orale : Une approche centrée sur l'apprenant ". ITL International Journal of Applied Linguistics, Volume, PP 65-79.

- Veltcheff, C. \& Hilton, S., (2003). L'évaluation en FLE, Paris, Hachette.

- Viallon, V.(2002). " Images et Apprentissage le Discours de l'images en Didactique des Langues". Condé sur Noireau, Vol.4, PP.207 - 240 .

- Watt, L.(2002). "L'apprentissage du Français Langue Etrangère Facilité par la Technologie". Mémoire de magistère, Université de Pretoria. 


\section{Number 57 , Part 2 , January, 2015}

- Weis, T; Benmayor, R.; O'Leary, C. \& Eynon, B.(2002). " Digital Technologies and Pedagogies, Journal of Social Justice, Volume (29), N.4, PP.153 - 167.

- Xiaohong, G. (2005) . "L'entrainement à l'utilisation des stratégies d'écoute vers un enseignement plus efficace de la compréhension orale en L2, Université Lumière Lyon2, France.

- Yuksel, P.(2011). "Using Digital Storytelling in Early Childhood Education : A Phenomenological Study of Teachers Experiences". The degree of Philosophy's doctor, The Middle East Technical University.

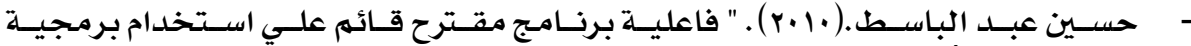

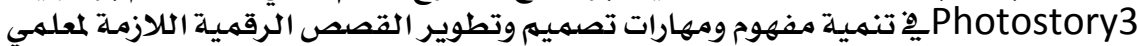

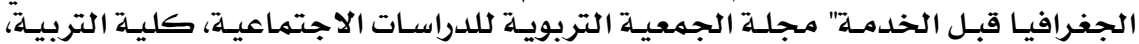

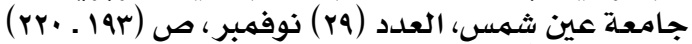

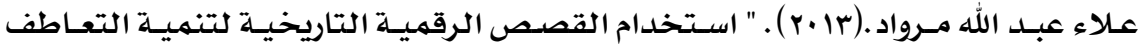

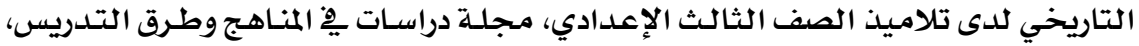

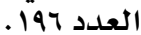

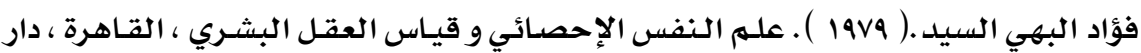

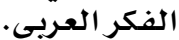

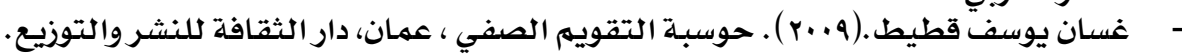

\section{溇溇溇潾溇}

